\title{
Triangular forms of subdiagonal algebras
}

\author{
Guoxing Ji, Tomoyoshi OHwada and Kichi-Suke SAITO*
}

(Received April 14, 1997)

\begin{abstract}
In this note, we study the triangular forms of subdiagonal algebras and discuss the triangular decomposition of subdiagonal algebras.
\end{abstract}

Key words: von Neumann algebra; expectation; subdiagonal algebra; nest algebra.

\section{Introduction and Preliminaries}

In [1], Arveson introduced the notion of subdiagonal algebras to give a unified approach to the theory of non-selfadjoint operator algebras. The algebra is not only a noncommutative analogue of weak $*$-Dirichlet algebras but also a generalization of the work of Helson-Lowdenslager in [4]. Thus, the algebra has many analytic properties as the algebra of generalized analytic functions. Several concrete examples were considered in [1]. Further, Loebl-Muhly [7] and Kawamura-Tomiyama [6] gave systematic examples of subdiagonal algebras from the theory of spectral subspaces determined by flows on a von Neumann algebra. We refer the readers to [1] for the elementary properties of subdiagonal algebras. On the other hand, the notion of nest algebras was introduced by Ringrose [8] to study the triangular forms for operators. The structure of nest algebras was studied by many authors and we refer the readers for the details to Davidson's book [3]. Our aim in this note is to study the triangular forms of subdiagonal algebras related to the theory of nest algebras.

At first, we start by giving the definition of subdiagonal algebras. Let $\mathcal{M}$ be a von Neumann algebra on a complex Hilbert space $\mathcal{H}$ and let $\Phi$ be a faithful normal positive linear map of $\mathcal{M}$ onto a von Neumann subalgebra $\mathfrak{D}$ of $\mathcal{M}$ which is idempotent, that is, let $\Phi$ be a faithful normal expectation of $\mathcal{M}$ onto $\mathfrak{D}$. A subalgebra $\mathfrak{A}$ of $\mathcal{M}$, containing $\mathfrak{D}$, is called a subdiagonal algebra in $\mathcal{M}$ with respect to $\Phi$ if

(i) $\mathfrak{A} \cap \mathfrak{A}^{*}=\mathfrak{D}$;

1991 Mathematics Subject Classification : Primary 46L10, Secondary 47D25.

*This work was supported in part by a Grand-in-Aid for Scientific Research from the Japanese Ministry of Education. 
(ii) $\Phi$ is multiplicative on $\mathfrak{A}$; and

(iii) $\mathfrak{A}+\mathfrak{A}^{*}$ is $\sigma$-weakly dense in $\mathcal{M}$.

The algebra $\mathfrak{D}$ is called the diagonal of $\mathfrak{A}$. We say that $\mathfrak{A}$ is a maximal subdiagonal algebra in $\mathcal{M}$ with respect to $\Phi$ in case $\mathfrak{A}$ is not properly contained in any other subalgebra of $\mathcal{M}$ which is subdiagonal with respect to $\Phi$.

Although subdiagonal algebras are not assumed to be $\sigma$-weakly closed in [1], the $\sigma$-weak closure of a subdiagonal algebra is again a subdiagonal algebra ([1, Remark 2.1.2]). In this note, we assume henceforth that our subdiagonal algebras are always $\sigma$-weakly closed.

Let $\mathfrak{A}$ be a subdiagonal algebra in $\mathcal{M}$ acting on a Hilbert space $\mathcal{H}$ and put $\mathfrak{A}_{0}=\{X \in \mathfrak{A}: \Phi(X)=0\}$. Then it is clear that $\mathfrak{A}_{0}$ is a $\sigma$-weakly closed two-sided ideal of $\mathfrak{A}$. For every $n \in \mathbb{N}$, we define the closed subspace $\mathcal{H}_{n}=\left[\mathfrak{A}_{0}^{n} \mathcal{H}\right]$, where $\mathfrak{A}_{0}^{n}=\left\{A_{1} A_{2} \cdots A_{n}: A_{i} \in \mathfrak{A}_{0}(1 \leq i \leq n)\right\}$ and, for a subset $\mathcal{S}$ of $\mathcal{H},[\mathcal{S}]$ is the closed linear span of $\mathcal{S}$. Further, we set $\mathcal{H}_{0}=\mathcal{H}$ and $\mathcal{H}_{\infty}=\bigcap_{n=0}^{\infty} \mathcal{H}_{n}$, respectively. For every $n \in \mathbb{N} \cup\{0, \infty\}$, let $P_{n}$ be the orthogonal projection from $\mathcal{H}$ onto $\mathcal{H}_{n}$. Then it is clear that the family $\left\{P_{n}: 0 \leq n \leq \infty\right\}$ of projections is a decreasing sequence in $\mathcal{M}$, such that $P_{n} \downarrow P_{\infty}$. Then we define the following notion.

Definition 1.1 Keep the notation as above. Then a subdiagonal algebra $\mathfrak{A}$ is said to be pure if $P_{\infty}=0$. Further, if $P_{1}=I$, then the subdiagonal algebra $\mathfrak{A}$ is called to be non-degenerate.

In this note, we investigate the purity of subdiagonal algebras. In $\S 2$, we consider the case that it is on a finite dimensional Hilbert space. If $\mathfrak{A}$ is a subdiagonal algebra in $\mathcal{M}$, more generally, $\mathfrak{A}$ is a subalgebra of $\mathcal{M}$ such that $\mathfrak{A}+\mathfrak{A}^{*}=\mathcal{M}$, then we show that $\mathfrak{A}$ is a nest subalgebra of $\mathcal{M}$ (Theorem 2.1). In particular, every subdiagonal algebra in the finite dimensional case is pure in the sense of Definition 1.1. In $\S 3$, we shall show that a subdiagonal algebra $\mathfrak{A}$ of $\mathcal{M}$ is pure if and only if $\mathfrak{A}$ is a nest subalgebra in $\mathcal{M}$ determined by $\left\{P_{n}: 0 \leq n \leq \infty\right\}$, that is, $\mathfrak{A}=\left\{A \in \mathcal{M}:\left(I-P_{n}\right) A P_{n}=0,0 \leq n \leq \infty\right\}$ (Theorem 3.2). Finally, we shall discuss the triangular decomposition of a subdiagonal algebra $\mathfrak{A}$ of $\mathcal{M}$ (Theorem 3.3).

\section{Finite dimensional case}

In this section, we assume that the Hilbert space $\mathcal{H}$ is finite dimensional. Then we shall study the structure of subdiagonal algebras in $\mathcal{M}$, more 
generally, subalgebras of $\mathcal{M}$ satisfying $\mathfrak{A}+\mathfrak{A}^{*}=\mathcal{M}$. At first we have the following theorem.

Theorem 2.1 Let $\mathfrak{A}$ be a subalgebra of $\mathcal{M}$, containing the identity, such that $\mathfrak{A}+\mathfrak{A}^{*}=\mathcal{M}$. Then $\mathfrak{A}$ is a nest subalgebra of von Neumann algebra $\mathcal{M}$.

To prove this, we need the following lemmas.

Lemma 2.2 Keep the assumptions as in Theorem 2.1. If $\mathfrak{A} \varsubsetneqq \mathcal{M}$, then there exists a non-trivial projection $P$ in $\mathcal{M}$ such that $P \in$ Lat $\mathfrak{A}$, where Lat $\mathfrak{A}$ is the lattice of all $\mathfrak{A}$-invariant subspaces of $\mathcal{H}$.

Proof. If $\mathcal{M}$ is not a factor, then we may take a non-trivial central projection $P$ in $\mathcal{M}$. Thus we may suppose that $\mathcal{M}$ is a factor. Since $\mathcal{H}$ is finite dimensional, $\mathcal{M}$ is a type $I_{k}$-factor for some $k$. By [5, Theorem 6.6.1], there exists a $*$-isomorphism $\Theta$ from $\mathcal{M}$ onto $\mathcal{B}(K)$ for a Hilbert space $\mathcal{K}$ with $\operatorname{dim} \mathcal{K}=k$. Since $\Theta(\mathfrak{A})$ is a proper subalgebra of $\mathcal{B}(K)$, by $[2$, Proposition 2.12], there is a non-trivial projection $Q$ in $\mathcal{B}(K)$ such that $Q \in \operatorname{Lat} \Theta(\mathfrak{A})$. Putting $P=\Theta^{-1}(Q), P$ has the desired property. This completes the proof.

Lemma 2.3 Keep the assumptions as in Theorem 2.1. If $P \in \mathcal{M} \cap$ Lat $\mathfrak{A}$, then $P \in \mathfrak{A}$.

Proof. If a projection $P \in \mathcal{M}$ and $\mathfrak{A}+\mathfrak{A}^{*}=\mathcal{M}$, then there exists an element $C \in \mathfrak{A}$ such that $P=C+C^{*}$. Considering the Hilbert space decomposion $\mathcal{H}=P \mathcal{H} \oplus P^{\perp} \mathcal{H}$, we can write the matrix representation of $C$ as follows:

$$
C=\left(\begin{array}{cc}
C_{11} & C_{12} \\
0 & C_{22}
\end{array}\right) .
$$

Since $P=P^{*}$, we have $C_{12}=0, C_{11}+C_{11}^{*}=I_{P \mathcal{H}}$ and $C_{22}+C_{22}^{*}=0$. Thus $C_{11}$ and $C_{22}$ are of the forms

$$
C_{11}=\frac{1}{2} I_{P \mathcal{H}}+i K_{1} \quad \text { and } \quad C_{22}=i K_{2}
$$

for some self-adjoint operators $K_{1} \in \mathcal{B}(P \mathcal{H})$ and $K_{2} \in \mathcal{B}\left(P^{\perp} \mathcal{H}\right)$. This implies that $C$ is a normal operator in $\mathfrak{A}$ with $\sigma(C)=\sigma\left(C_{11}\right) \cup \sigma\left(C_{22}\right)$, where $\sigma(A)$ is the spectrum of an operator $A$. Since $\sigma\left(C_{11}\right) \subset\left\{\frac{1}{2}+i \lambda: \lambda \in\right.$ $\mathbb{R}\}$ and $\sigma\left(C_{22}\right) \subset\{i \lambda: \lambda \in \mathbb{R}\}$ respectively, we have $\sigma\left(C_{11}\right) \cap \sigma\left(C_{22}\right)=\emptyset$. 
We now choose two bounded open subsets $\Omega_{1}$ and $\Omega_{2}$ in $\mathbb{C}$ with the following properties:

(1) $\sigma\left(C_{11}\right) \subset \Omega_{1}$ and $\sigma\left(C_{22}\right) \subset \Omega_{2}$

(2) $\bar{\Omega}_{1} \cap \bar{\Omega}_{2}=\emptyset$; and

(3) $\left(\bar{\Omega}_{1} \cup \bar{\Omega}_{2}\right)^{c}$ is connected.

Put $f=\chi_{\Omega_{1}}$, where $\chi_{\Omega_{1}}$ is the characteristic function of $\Omega_{1}$. By the functional calculus and [9, Theorem 13.7], we have $f(C)=P \in \mathfrak{A}$. This completes the proof.

Proof of Theorem 2.1. We may assume that $\mathfrak{A} \neq \mathcal{M}$. By Lemma 2.2, there exists a maximal nest $\mathcal{N}$ in $\mathcal{M} \cap$ Lat $\mathfrak{A}$. We set $\mathcal{N}=\left\{Q_{k}\right\}_{k=0}^{n}$ satisfying

$$
0=Q_{0}<Q_{1}<Q_{2}<\cdots<Q_{n}=I .
$$

Since $\mathcal{N} \subset \mathfrak{A}$ by Lemma 2.3, it is enough to prove that $\mathfrak{A}=\mathcal{M} \cap \operatorname{alg} \mathcal{N}$. It is trivial that $\mathfrak{A} \subseteq \mathcal{M} \cap \operatorname{alg} \mathcal{N}$. Put $E_{k}=Q_{k}-Q_{k-1}(k=1,2, \ldots, n)$. By the maximality of $\mathcal{N}$ and Lemma 2.2, we have $E_{k} \in \mathfrak{A}$ and $E_{k} \mathfrak{A} E_{k}=E_{k} \mathcal{M} E_{k}$. Let $T \in \mathcal{M} \cap \operatorname{alg} \mathcal{N}$. Then $E_{k} T E_{j}=0$ for $k>j$. Since $\mathfrak{A}+\mathfrak{A}^{*}=\mathcal{M}$, we take $A$ and $B$ in $\mathfrak{A}$ such that $A+B^{*}=T$. By a simple calculation, then we have $E_{k} T E_{j} \in \mathfrak{A}(k, j=1,2, \ldots, n)$ and so $T \in \mathfrak{A}$. This completes the proof.

Let $\mathfrak{A}$ be a subdiagonal algebra in $\mathcal{M}$ with respect to $\Phi$. As in the proof of Theorem 2.1, there exists a finite nest $\mathcal{N}=\left\{Q_{k}\right\}_{k=1}^{n}$ in $\mathcal{M}$ such that $\mathfrak{A}=\mathcal{M} \cap \operatorname{alg} \mathcal{N}$. Further, we easily show that $\Phi(X)=\sum_{k=1}^{n} E_{k} X E_{k}$ for every $X \in \mathcal{M}$ and $\mathfrak{A}_{0}^{n}=0$. Thus we have the following corollary.

Corollary 2.4 If $\mathfrak{A}$ is a subdiagonal algebra in $\mathcal{M}$ with respect to $\Phi$, then $\mathfrak{A}$ is a nest subalgebra of $\mathcal{M}$ with a finite nest, in particular, $\mathfrak{A}$ is pure in the sense of Definition 1.1 .

In [10, Theorem 2], the third author and Watatani showed that if $\mathfrak{D}$ is a subfactor of a finite dimensional factor $\mathcal{M}$, then there exist no maximal subdiagonal algebras of $\mathcal{M}$ with diagonal $\mathfrak{D}$ unless $\mathfrak{D}=\mathcal{M}$. More generally, we have the following corollary.

Corollary 2.5 Let $\mathcal{M}$ be a finite dimensional von Neumann algebra and let $\mathfrak{D}$ be a subfactor of $\mathcal{M}$. Then there exist no subdiagonal algebras in $\mathcal{M}$ with diagonal $\mathfrak{D}$ unless $\mathfrak{D}=\mathcal{M}$.

Proof. $\quad$ Let $\mathfrak{A}$ be a subdiagonal algebra in $\mathcal{M}$ with diagonal $\mathfrak{D}$. By Theo- 
rem $2.1, \mathfrak{A}$ is a nest subalgebra of $\mathcal{M}$ with a nest $\mathcal{N}$ in $\mathfrak{D} \cap \mathfrak{D}^{\prime}$. Since $\mathfrak{D}$ is a subfactor of $\mathcal{M}$, we have $\mathcal{N}=\{0, I\}$ and so $\mathfrak{D}=\mathfrak{A}=\mathcal{M}$. This completes the proof.

\section{Pure subdiagonal algebras}

In this section, we investigate the purity of a subdiagonal algebra in $\mathcal{M}$ acting on an infinite dimensional Hilbert space $\mathcal{H}$. Note that $P_{n}$ is the orthogonal projection from $\mathcal{H}$ onto $\left[\mathfrak{A}_{0}^{n} \mathcal{H}\right]$ defined in $\S 1$. At first we have the following proposition.

Proposition 3.1 For every $n$ in $\mathbb{N} \cup\{0, \infty\}, P_{n}$ is a central projection of $\mathfrak{D}$.

Proof. Since $\left\{P_{n}: 0 \leq n \leq \infty\right\} \subseteq$ Lat $\mathfrak{A} \subseteq \mathfrak{D}^{\prime}$, it is enough to prove that $\left\{P_{n}: 0 \leq n \leq \infty\right\} \subseteq \mathfrak{D}$. To do this, we need only to prove that $\Phi\left(P_{n}\right)=P_{n}$ for every $n=0,1,2, \ldots, \infty$. Putting $E_{n}=P_{n} \ominus P_{n+1}(n \geq 0)$, then we have

$$
I=\left(\sum_{n=0}^{\infty} \oplus E_{n}\right) \oplus P_{\infty} .
$$

Since $\mathfrak{A}_{0} \mathcal{H}_{n} \subseteq \mathcal{H}_{n+1}$, we have $E_{n} \mathfrak{A}_{0} E_{n}=0$. Since $X-\Phi(X) \in \mathfrak{A}_{0}$ for every $X \in \mathfrak{A}$ and $\mathfrak{A}+\mathfrak{A}^{*}$ is $\sigma$-weakly dense in $\mathcal{M}$, it follows that

$$
E_{n} X E_{n}=E_{n} \Phi(X) E_{n} \quad(n \geq 0)
$$

for every $X \in \mathcal{M}$. In particular,

$$
E_{n} \Phi\left(E_{k}\right) E_{n}=\left\{\begin{array}{rr}
0, & n \neq k \\
E_{n}, & n=k .
\end{array}\right.
$$

Thus we have

$$
\begin{aligned}
\Phi\left(E_{k}\right) & =\sum_{n=0}^{\infty} E_{n} \Phi\left(E_{k}\right)+P_{\infty} \Phi\left(E_{k}\right) \\
& =\sum_{n=0}^{\infty} E_{n} \Phi\left(E_{k}\right) E_{n}+P_{\infty} \Phi\left(E_{k}\right) P_{\infty} \\
& =E_{k}+P_{\infty} \Phi\left(E_{k}\right) P_{\infty}
\end{aligned}
$$

which implies that $\Phi\left(E_{k}\right) \geq E_{k}(k \geq 0)$. Since $\Phi$ is faithful and idempotent, $\Phi\left(E_{k}\right)=E_{k}(k \geq 0)$ and so $\Phi\left(P_{k}\right)=P_{k}(k \geq 0)$. This completes the proof. 
Our goal in this section is the following theorem.

Theorem 3.2 Let $\mathfrak{A}$ be a subdiagonal algebra in $\mathcal{M}$. Then $\mathfrak{A}$ is pure if and only if there exists a finite or infinite decreasing nest $\mathcal{N}=\left\{Q_{n}: 0 \leq n \leq \infty\right\}$ in $\mathcal{M}$ such that $\mathfrak{A}=\mathcal{M} \cap \operatorname{alg} \mathcal{N}$.

Proof. $\quad(\Longrightarrow)$ Suppose that $\mathfrak{A}$ is pure. Put $\mathcal{N}=\left\{P_{n}: 0 \leq n \leq \infty\right\}$. Since $P_{\infty}=0$, as in the proof of Proposition 3.1, we have $E_{n} \mathcal{M} E_{n} \subseteq \mathfrak{D} \subseteq \mathfrak{A}$ for every $n \geq 0$ and

$$
\Phi(X)=\sum_{n=0}^{\infty} E_{n} X E_{n} \quad(X \in \mathcal{M}) .
$$

Since $P_{n} \mathcal{H}\left(=\left[\mathfrak{A}_{0}^{n} \mathcal{H}\right]\right)$ is $\mathfrak{A}$-invariant, it is clear that $\mathfrak{A} \subseteq \mathcal{M} \cap \operatorname{alg} \mathcal{N}$.

Conversely, if $T \in \mathcal{M} \cap \operatorname{alg} \mathcal{N}$, then it is clear that $E_{j} T E_{k}=0$ for $j<k$ and $T=\sum_{j \geq k} E_{j} T E_{k}$ in the $\sigma$-weak topology. We next prove that $E_{j} T E_{k} \in \mathfrak{A}$. We know $E_{k} T E_{k} \in \mathfrak{D}(k \geq 1)$ by Proposition 3.1. When $j>k$, there are two nets $\left\{A_{\alpha}\right\}$ and $\left\{B_{\alpha}\right\}$ in $\mathfrak{A}$ such that $T=\lim _{\alpha}\left(A_{\alpha}+B_{\alpha}^{*}\right)$ $\sigma$-weakly. Since $\mathfrak{A} \subseteq \mathcal{M} \cap \operatorname{alg} \mathcal{N}$ we have $E_{j} B_{\alpha}^{*} E_{k}=0$ for $j>k$. Then $E_{j} T E_{k}=\lim _{\alpha} E_{j} A_{\alpha} E_{k} \in \mathfrak{A}$ for $j>k$. Thus $T \in \mathfrak{A}$ and we show that $\mathfrak{A}=\mathcal{M} \cap \operatorname{alg} \mathcal{N}$.

$(\Longleftarrow)$ Suppose that there exists a decreasing nest $\mathcal{N}=\left\{Q_{n}: 0 \leq n \leq\right.$ $\infty\}$ in $\mathcal{M}$ such that $\mathfrak{A}=\mathcal{M} \cap \operatorname{alg} \mathcal{N}$. Thus we have $\mathfrak{D}=\mathcal{N}^{\prime} \cap \mathcal{M}$ and $\Phi(X)=\sum_{n=0}^{\infty} F_{n} X F_{n}$, where $F_{n}=Q_{n} \ominus Q_{n+1}(n \geq 0)$. Since $\mathfrak{A}_{0}$ may be regarded as the strictly lower triangular algebra with entries in $\mathcal{M}$, we have

$$
\left[\mathfrak{A}_{0}^{n} \mathcal{H}\right] \subseteq Q_{n} \mathcal{H}
$$

which implies that $\bigcap_{n=0}^{\infty}\left[\mathfrak{A}_{0}^{n} \mathcal{H}\right]=\{0\}$. Thus $\mathfrak{A}$ is pure. This completes the proof.

In general, taking a projection $E \in \mathfrak{D}$, we define the faithful normal expectation $\Phi_{E}$ from $E \mathcal{M} E$ onto $E \mathfrak{D} E$ by $\Phi_{E}(E X E)=E \Phi(X) E$. Note that $E \mathfrak{A} E$ is a subdiagonal algebra in $E \mathcal{M} E$ with respect to $\Phi_{E}$.

If $P_{\infty} \neq 0$, then we have $\left[\mathfrak{A}_{0} P_{\infty} \mathcal{H}\right]=P_{\infty} \mathcal{H}$. Since $P_{\infty} \in \mathfrak{D}, P_{\infty} \mathfrak{A} P_{\infty}$ is a non-degenerate subdiagonal algebra in $P_{\infty} \mathcal{M} P_{\infty}$ with respect to $\Phi_{P_{\infty}}$. On the other hand, $P_{\infty}^{\perp} \mathfrak{A} P_{\infty}^{\perp}$ is a pure subdiagonal algebra in $P_{\infty}^{\perp} \mathcal{M} P_{\infty}^{\perp}$ with respect to $\Phi_{P_{\infty}^{\perp}}$ and $P_{\infty} \mathfrak{A} P_{\infty}^{\perp}=P_{\infty} \mathcal{M} P_{\infty}^{\perp}$. Thus we have a decomposition of a subdiagonal algebra. Moreover, since $P_{\infty} \mathfrak{A}^{*} P_{\infty}$ is also a subdiagonal algebra in $P_{\infty} \mathcal{M} P_{\infty}$, we may also consider the decomposition of $P_{\infty} \mathfrak{A}^{*} P_{\infty}$. 
Then we easily have the following decomposition.

Theorem 3.3 Let $\mathfrak{A}$ be a subdiagonal algebra in $\mathcal{M}$ with respect to $\Phi$. Then there exist three mutually orthogonal projections $E_{1}, E_{2}$ and $E_{3}$ in $\mathfrak{D}$ such that

(i) $E_{1}+E_{2}+E_{3}=I$ and $E_{1}, E_{1}+E_{2} \in$ Lat $\mathfrak{A}$,

(ii) $\mathfrak{A}$ has the following matrix decomposition:

$$
\mathfrak{A}=\left(\begin{array}{ccc}
\mathfrak{A}_{11} & \mathfrak{A}_{12} & \mathfrak{A}_{13} \\
0 & \mathfrak{A}_{22} & \mathfrak{A}_{23} \\
0 & 0 & \mathfrak{A}_{33}
\end{array}\right),
$$

where $\mathfrak{A}_{11}^{*}$ (resp. $\mathfrak{A}_{33}$ ) is a pure subdiagonal algebra in $E_{1} \mathcal{M} E_{1}$ (resp. $\left.E_{3} \mathcal{M} E_{3}\right), \mathfrak{A}_{22}$ and $\mathfrak{A}_{22}^{*}$ are non-degenerate subdiagonal algebras in $E_{2} \mathcal{M} E_{2}$ and $\mathfrak{A}_{j k}=E_{j} \mathcal{M} E_{k}$ for $j<k$.

\section{References}

[1] Arveson W.B., Analyticity in operator algebras. Amer. J. Math. 89 (1967), 578642.

[2] Azoff E.A., On finite rank operators and preannihilators. Mem. Amer. Math. Soc. 357 (1986).

[3] Davidson K.R., Nest algebras. Pitman Research Notes in Mathematics Series, vol. 191, Longman Scientific and Technical Pub. Co., London, New York, 1988.

[4] Helson H. and Lowdenslager D., Prediction theory and Fourier series in several variables. Acta Math. 99 (1958), 165-202.

[5] Kadison R.V. and Ringrose J. R., Fundamentals of the theory of operator algebras II. Academic Press, 1986.

[6] Kawamura S. and Tomiyama J., On subdiagonal algebras associated with flows in opertor algebras. J. Math. Soc. Japan 29 (1977), 73-90.

[7] Loebl R. and Muhly P.S., Analyticity and flows in von Neumann algebras. J. Funct. Anal. 29 (1978), 214-252.

[8] Ringrose J., On some algebras of operators. Proc. London Math. Soc. 15 (1965), 61-83.

[9] Rudin W., Real and complex analysis. the third edition, McGraw-Hill, New York, 1987.

[10] Saito K-S. and Watatani Y., Subdiagonal algebras for subfactors II. Canad. Math. Bull. 40 (1997), 254-256. 


\author{
Guoxing Ji \\ Department of Mathematical Science \\ Graduate School of Science and Technology \\ Niigata University \\ Niigata, 950-2181 Japan \\ Current address: \\ Department of Mathematics \\ Shaanxi Normal University \\ Xian, 710062, Shaanxi \\ P.R. China \\ Tomoyoshi Ohwada \\ Department of Mathematical Science \\ Graduate School of Science and Technology \\ Niigata University \\ Niigata, 950-2181 Japan \\ E-mail: ohwada@math.sc.niigata-u.ac.jp \\ Kichi-Suke Saito \\ Department of Mathematics \\ Faculty of Science, Niigata University \\ Niigata, 950-2181, Japan \\ E-mail: saito@math.sc.niigata-u.ac.jp
}

